\title{
eJRIEPS
}

Ejournal de la recherche sur l'intervention en éducation physique et sport

38 | 2016

Varia

\section{Avril 2016 Journée internationale d'étude René Deleplace}

Didier Barthès, Gilles Uhlrich, Marie-Paule Poggi, Mathilde Musard et Nathalie Wallian

\section{(2) OpenEdition}

Journals

Édition électronique

URL : http://journals.openedition.org/ejrieps/879

DOI : 10.4000/ejrieps.879

ISSN : 2105-0821

Éditeur

ELLIADD

\section{Référence électronique}

Didier Barthès, Gilles Uhlrich, Marie-Paule Poggi, Mathilde Musard et Nathalie Wallian, « Avril 2016 Journée internationale d'étude René Deleplace », eJRIEPS [En ligne], 38 | 2016, mis en ligne le 01 avril 2016, consulté le 24 septembre 2020. URL : http://journals.openedition.org/ejrieps/879 ; DOI : https:// doi.org/10.4000/ejrieps.879

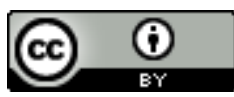

La revue eJRIEPS est mise à disposition selon les termes de la Creative Commons Attribution 4.0 International License. 


\section{eJRIEPS 38}

Les propositions de manuscrits sont à envoyer à :

ejrieps@orange.fr

Ce numéro 38 d'eJRIEPS présente des articles liés à deux manifestations scientifiques dont les productions comptent pour notre champ : la journée René Deleplace et la journée Alain Durey.

Vous trouverez quatre contributions proposées dans le cadre de la 3ème Journée internationale d'étude René Deleplace qui s'est déroulée le 21 mars 2014 à Bordeaux et deux textes qui rendent compte des interventions des conférenciers invités à la septième journée Alain Durey qui s'est déroulée à Créteil le 19 mars 2015.

Cet édito a donc été rédigé à plusieurs mains en collaboration avec six éditeurs associés. Nous remercions Didier Barthès et Gilles Uhlrich qui ont œuvré à rendre compte de la Journée Deleplace mais également Fabienne Brière-Guenoun, Serge Éloi, Alain Mouchet et Jean-François Robin à l'initiative de la restitution des travaux produits dans le cadre de la journée Alain Durey. Tous sont les artisans de ces différentes manifestations scientifiques qui donnent vie et aident à l'épanouissement de notre champ scientifique. 


\section{Avril 2016 Journée internationale d'étude René Deleplace}

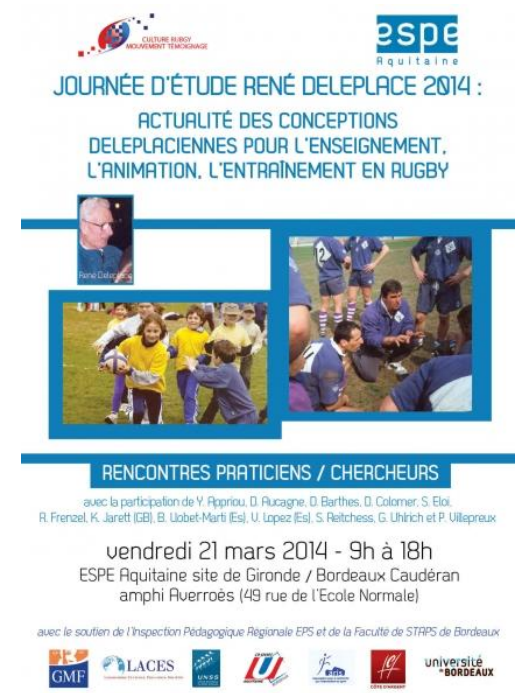

Ce numéro d'eJRIEPS est consacré à un compte-rendu de la Journée internationale d'étude René Deleplace qui s'est déroulée le 21 mars 2014 à Bordeaux. Comme chacun sait, René Deleplace a fortement contribué à la théorisation du jeu en rugby et en sports collectifs depuis les années 50. Cette troisième édition des Journées Deleplace vise à rendre compte de l'influence des travaux de ce théoricien tant sur les pratiques professionnelles actuelles (enseignement, animation, entraînement) que sur les recherches scientifiques, et de les situer au regard des courants internationaux technologiques et didactiques des sports collectifs. C'est sous l'impulsion de L'association "Culture Rugby de Mouvement Témoignage", adossée à l'INSEP et la Fédération Française de Rugby, de l'ESPE d'Aquitaine, de l'équipe Vie Sportive du Laboratoire Cultures, Education, Sociétés, et du département STAPS de I'Université de Bordeaux qu'un espace de rencontre a été offert à des praticiens et des chercheurs intéressés par la modélisation technologique et le traitement didactique du rugby ou des sports collectifs.

Les travaux présentés interrogent les évolutions historiques contemporaines des pratiques et des recherches, les différenciations liées aux secteurs sociaux de pratique, les modalités de transmission-appropriation du rugby et des sports collectifs. Cette journée intègre également des préoccupations pluridisciplinaires, mais aussi les attentes comparatistes (entre secteurs sociaux de pratique et entre nations) et le développement de collaborations et de retentissements internationaux. En effet, dans ce numéro, aux 


\section{eJRIEPS 38 avril 2016}

côtés des auteurs français (D. Bouthier, G. Uhlrich, S. Eloi), des chercheurs venus des Etats-Unis (Stephen Harvey), d'Australie (Kendall Jarrett) ou encore d'Espagne (V. LopezRos) apportent leur contribution confirmant ainsi le principe d'une collaboration internationale durable est engagée.

Le premier texte, intitulé Initiation et perfectionnement en sports collectifs: développer la pertinence des prises de décision en jeu, en lien avec les autres composantes de l'action rédigé par Daniel Bouthier vise à présenter dans ses déterminants socio-historique l'approche appelée « tactico-technique ». Prenant appui sur les travaux des principaux concepteurs de cette approche, l'auteur montre de quelle manière cette dernière a permis d'opérer une véritable rupture épistémologique dans la conception et la formation aux sports collectifs en proposant de partir de l'analyse des rapports d'opposition entre attaque et défense qui contextualisent et donnent sens à la technique considérée comme seconde. II ouvre la voie à un modèle d'analyse heuristique valorisant l'évolution et la complexification des rapports d'opposition.

Dans Développer l'intelligence tactique en jeu par la pédagogie des modèles de décision, dans la perspective francophone, Gilles Uhlrich et Serge Éloi s'intéressent aux procédés utiles au fonctionnement d'une pédagogie des modèles de décision tactique soucieuse de se distancier d'une forme techno centrée des situations d'apprentissage. Les auteurs décrivent avec finesse et précision les dimensions essentielles de l'intelligence tactique (Deleplace, 1979, 1983) associant avec justesse connaissances scientifiques et expérience pratique afin de donner du sens à la mise en place de dispositifs d'apprentissage.

Les deux derniers textes témoignent d'une ouverture des réflexions à l'international. Victor López-Ros signe un article intitulé El «modelo integrado técnico-táctico 》 de enseñanza deportiva. Origen, contextualización y características metodológicas dans lequel il s'intéresse à la façon dont le « Modèle Intégré Technique-Tactique » a été conçu et développé en Espagne. L'auteur insiste sur la dimension socio-constructiviste de cette approche qui favorise des apprentissages significatifs pour les élèves. II montre de quelle manière en faire un outil utile à un enseignement compréhensif.

Enfin, pour clore ce numéro, Kendall Jarrett et Stephen Harvey, dans Similar, but not the same: Comparing the game based approaches of Teaching Games for Understanding (TGfU) and Game Sense proposent une analyse fine des différences et des points communs entre deux approches, «TGfU » et « Game Sense » dont les auteurs 


\section{eJRIEPS 38 avril 2016}

discutent l'intérêt pour les enseignants et les entraîneurs, à partir de l'étude de la littérature mais également en prenant appui sur l'analyse de leçons.

Ce numéro spécial intéressera aussi bien les néophytes de la pensée de Deleplace que les spécialistes de l'enseignement des sports collectifs déjà aguerris aux travaux de cet auteur qui découvriront de nouvelles pistes de réflexions et d'analyse. L'autre défi relevé par les chercheurs ayant œuvré à ce numéro est d'avoir uni dans un même projet chercheurs et praticiens. Que vous soyez, étudiants, praticiens, formateurs ou chercheurs, vous trouverez dans cette lecture l'occasion d'interroger, d'éclairer ou encore de fonder vos pratiques d'enseignement des sports collectifs.

\section{Didier Barthès et Gilles Uhlrich (Editeurs associés) \\ Marie-Paule Poggi, Mathilde Musard \\ et Nathalie Wallian (Editrices scientifiques eJRIEPS)}

\section{Avril 2016 Journée Alain Durey}
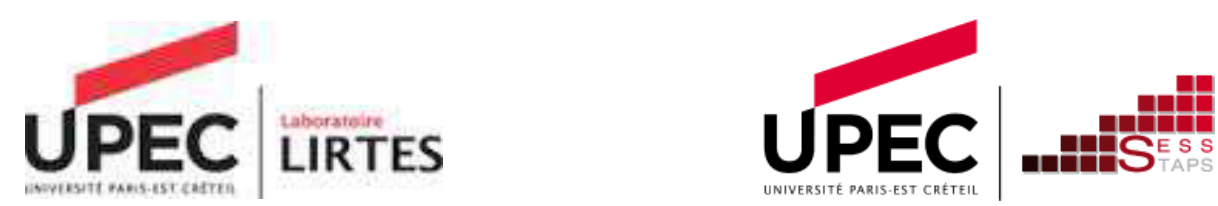

Les deux textes proposés dans ce numéro rendent compte des interventions de deux des conférenciers, Anne Jorro et Marc Cizeron, lors de la septième journée Alain Durey, qui s'est déroulée à Créteil le 19 mars 2015. Centrée sur la thématique des « gestes de métier des intervenants éducatifs », cette journée d'étude visait à mettre en débat l'usage de ces concepts dans les recherches actuelles portant sur « les gestes de métier » dans le domaine des pratiques éducatives et en particulier de l'enseignement. L'analyse des gestes de métier ou des gestes professionnels occupe en effet une place centrale dans de nombreuses recherches actuelles en sciences humaines et sociales. Au-delà de son caractère polysémique, le concept de geste permet de décrire l'activité de l'intervenant éducatif au regard de ses motifs d'agir, de ses préoccupations, de ses savoirs 


\section{eJRIEPS 38 avril 2016}

d'expérience, des enjeux de savoirs, des éléments de contexte, de l'activité d'apprentissage des élèves, des dimensions socioculturelles partagées au sein de la profession... Plusieurs questions ont orienté les interventions de cette journée d'étude :

- Quelle définition est donnée au concept de « geste professionnel » et/ou « geste de métier »?

- Quels choix théoriques et méthodologiques sont effectués pour analyser ces gestes ?

- Comment sont pensées les articulations entre gestes spécifiques et gestes génériques ?

- Quelles perspectives de transformation des pratiques, d'évolution des dispositifs de formation et de développement de l'activité des intervenants éducatifs sont envisagées ?

Le premier texte, celui d'Anne Jorro, s'intéresse aux postures et aux gestes professionnels de formateurs dans des situations d'accompagnement professionnel. Prenant appui sur une recherche collaborative conduite avec 13 formateurs du premier degré autour de 10 situations d'analyse de pratiques, il rend compte de la place de la corporéité dans l'activité du formateur rattachée au concept de posture. Selon l'approche développée par l'auteure, qui emprunte ses fondements à l'ethnologie, la phénoménologie et l'anthropologie historique, elle « indique la manière dont l'acteur se positionne dans un contexte social donné " et " extériorise une intention d'agir qui se manifestera non seulement dans l'action auprès d'autrui mais aussi à travers des actes précis que sont les gestes professionnels ». Les principaux résultats de cette recherche mettent en évidence cinq postures caractérisant l'activité du formateur, qui renvoient à l'identification d'intentions d'agir spécifiques de l'accompagnement professionnel d'enseignants débutants, celles de pisteur, de contradicteur, de traducteur, de médiateur et de régulateur. Ces postures sont étroitement articulées aux gestes professionnels, comme en témoigne l'association des gestes de désignation, explicatif et d'institutionnalisation à la posture de traducteur ou encore l'association des gestes de questionnement et de problématisation à la posture de contradicteur. Au-delà, la recherche présentée questionne l'existence de telles postures dans l'accompagnement des enseignants ayant du métier dans un domaine où le genre professionnel du formateur n'est pas institué. Elle souligne également l'intérêt de s'intéresser à l'intercorporéité, à travers l'identification des postures et des gestes professionnels, pour favoriser le développement professionnel des formateurs.

Le second texte est proposé par Marc Cizeron et il offre une réflexion novatrice sur le guidage des apprentissages des élèves. II porte sur la question des connaissances qui permettent à l'enseignant d'intervenir auprès des élèves pour guider leurs apprentissages, 


\title{
eJRIEPS 38 avril 2016
}

tout particulièrement dans les séquences de la leçon qualifiées de « supervision active ». Adossé à une conception gestaltiste des conduites motrices, le cadre théorique est présenté ainsi que les positions méthodologiques afférentes. II analyse les conduites motrices des élèves en EPS afin de guider leurs apprentissages. Sa complétude permet d'en apprécier toute la qualité. La partie de présentation des résultats ainsi que leur discussion est centrée sur les résultats les plus saillants et exemplaires, qui ont été obtenus à partir de trois études conduites en gymnastique, en natation et en danse. Marc Cizeron postule que le terme de geste est utilisé pour traduire l'idée que l'action du maître est une action de communication qui adhère à une culture partagée. Pour lui, le geste essentiel devient un geste d'intelligibilité, de compréhension partagée entre l'enseignant et l'élève.

Anne Jorro et Marc Cizeron contribuent à éclairer la thématique des « gestes de métier des intervenants éducatifs ». Ils participent de ce mouvement qui souhaite mieux comprendre comment le métier se fait tant du point de vue des intentions que des actions et des connaissances des enseignants pour guider les apprentissages.

\author{
Fabienne Brière-Guenoun, Serge Éloi, Alain Mouchet \\ et Jean-François Robin (Editeurs associés) \\ Marie-Paule Poggi, Mathilde Musard \\ et Nathalie Wallian (Editrices scientifiques eJRIEPS)
}

